\title{
Transarterial chemoembolization for hepatocellular carcinoma: an evidence-based review of its place in therapy
}

This article was published in the following Dove Press journal:

Journal of Hepatocellular Carcinoma

2 September 2015

Number of times this article has been viewed

\section{Ji Hye Jang \\ Jin-Woo Lee \\ ji Taek Hong \\ Young-Joo Jin}

Division of Gastroenterology and Hepatology, Inha University School of Medicine and Hospital, Incheon, Republic of Korea
Correspondence: Jin-Woo Lee Division of Gastroenterology and Hepatology, Inha University School of Medicine and Hospital, 27 Inhang-ro, Jung-Gu, Incheon 400-7I I, Republic of Korea

Tel +82328902548

Fax +82 328826578

Email jin@inha.ac.kr
Abstract: Transarterial chemoembolization (TACE) is the standard treatment for patients with Barcelona Clinic Liver Cancer-intermediate stage hepatocellular carcinoma (HCC). The concept of drug-eluting bead TACE builds on the rationale of intratumoral drug delivery, and drug-eluting bead TACE has been shown to provide consistent and reliable results and to significantly diminish systemic drug exposure, liver toxicity, and drug-related adverse events as compared with conventional TACE. Based on the belief that combinations of TACE and other local or systemic therapies have several theoretical advantages, many clinical trials have been conducted to evaluate the effectiveness of TACE in combination with local treatment such as radiofrequency ablation or radiotherapy, and systemic therapy such as sorafenib or another molecular therapy. TACE has also been used as a preoperative adjuvant chemotherapy in patients with HCC to improve survival and as a bridging therapy before liver transplantation to downstage HCC. In the present evidence-based review, the authors summarize the current status of these transcatheter arterial embolic therapies in HCC.

Keywords: transarterial chemoembolization (TACE), hepatocellular carcinoma (HCC), evidence-based review

\section{Introduction}

Hepatocellular carcinoma (HCC) remains a global health problem and is currently ranked as the third leading cause of cancer-related death. ${ }^{1,2}$ The majority of cases arise due to cirrhotic liver, in which many etiologic factors are involved. While treatment options for HCC such as surgical resection, liver transplantation, and ablative therapies provide a chance of cure, these modalities are limited and often ineffective, because HCC is usually diagnosed at an advanced stage. Therefore, advanced stage presentation, poor liver function, severe comorbidities, and the limitation of available donors make it difficult to receive curative treatments for HCC. Accordingly, palliative therapies such as transcatheter arterial embolic therapies, radiation therapy, and systemic chemotherapies provide treatment options.

Transarterial chemoembolization (TACE) has been widely performed and has become the current standard therapy for large or multinodular HCC with a relatively preserved liver function, the absence of cancer-related symptoms, and no evidence of vascular invasion or extrahepatic spread, which is classified as intermediate stage according to the Barcelona Clinic Liver Cancer (BCLC) staging system. ${ }^{1}$

TACE involves the injection of an embolic agent into the tumor-feeding arteries to block its major nutrient source, and results in ischemic necrosis of the targeted tumor. The European Association for the Study of the Liver (EASL) guidelines recommend 
TACE for unresectable HCC with BCLC-B intermediate stage. $^{3}$ Drug-eluting bead TACE (DEB-TACE) represents a novel drug delivery/embolization system, and was developed to deliver higher doses of chemotherapeutic agents over longer drug-tumor contact times. ${ }^{2}$ It has been shown that in $\mathrm{HCC}$ patients, TACE with drug-eluting microparticles (DC Bead $^{\circledR}$ [BTG International Ltd, London, UK]) achieves higher intratumoral but lower systemic concentrations of doxorubicin than conventional TACE (cTACE) and reduces liver toxicity. ${ }^{2,4}$

TACE has also been performed as a preoperative chemotherapy in $\mathrm{HCC}$ to improve survival and as a bridging therapy before liver transplantation for HCC downstaging. ${ }^{5}$ On the other hand, based on the concept that TACE in combination with other local or systemic therapies has several theoretical advantages, many clinical trials have been conducted to evaluate the effectiveness of TACE plus another local treatment such as radiofrequency ablation (RFA) and radiotherapy, or systemic molecular target therapy. However, outcomes of clinical trials conducted on this topic are conflicting, and not all patients with intermediate-stage HCC are suitable for TACE; in such patients, other treatments such as sorafenib should be considered.

In this review, we summarize, on the basis of existing evidence, the current status of TACE in HCC and discuss related topics including DEB-TACE, bridging therapies to surgical resection, and combination therapies.

\section{Indications and contraindications for TACE}

The EASL guidelines recommend TACE for unresectable, Child-Pugh A or B multiple HCC with no vascular invasion. ${ }^{3}$ Liver function is a critical component of careful patient selection for TACE, which is discouraged in patients with decompensated liver disease, advanced liver dysfunction, macroscopic invasion, or extrahepatic spread. ${ }^{1}$

According to the BCLC staging system, HCC patients with portal vein tumor thrombosis (PVTT), BCLC stage $\mathrm{C}$, can receive only sorafenib target therapy. However, for patients with advanced HCC with vascular invasion or extrahepatic metastases, median survival for sorafenib is short (only 6.5 months) in Asia. ${ }^{6}$ Meta-analysis of eight controlled trials involving $1601 \mathrm{HCC}$ patients showed that TACE significantly improved 6-month (hazard ratio [HR], $0.41 ; 95 \%$ confidence interval $[\mathrm{CI}]=0.32-0.53 ; z, 6.28$; $P=0.000)$ and 1 -year (HR, 0.44; 95\% CI $=0.34-0.57 ; z, 6.22$; $P=0.000$ ) overall survivals (OSs) of patients with PVTT vs conservative treatment, which means that TACE has the potential to improve survival and is safe for advanced HCC with PVTT, even with main portal vein obstruction. ${ }^{7}$ Therefore, TACE may be extended in some patients with BCLC stage C.

The indications for TACE are still debated and vary between centers and countries. Furthermore, rules for its discontinuance are even less clear, and data on this subject are limited, mainly because there are no well-defined criteria for TACE failure and refractoriness. The need to define TACE-refractory HCC is particularly important in the strategy of repeating multiple sessions of TACE for residual viable tumors or newly developed lesions on an on-demand basis. One study of 264 patients with intermediate-stage HCC who underwent TACE showed that progression or the need for three sessions of TACE within the first 6 months might be predictive of TACE refractoriness. ${ }^{8}$ The Japan Society of Hepatology defined TACE failure or refractoriness as ineffective response (viable $\mathrm{HCC}$ lesions $>50 \%$ ) even after two or more consecutive TACE sessions, ${ }^{8}$ which is supported by the finding that the OS of patients treated with sorafenib induced by TACE failure or refractoriness without extrahepatic spread or vascular invasion was greater in patients that received $\leq 2$ consecutive ineffective TACE procedures. ${ }^{9}$ Furthermore, the results of this study support the definition of TACE failure or refractoriness as proposed by the Liver Cancer Study Group of Japan. ${ }^{10}$

Meanwhile, several authors have proposed that Assessment for Retreatment with TACE score (ART score), which is assessed using an increase in aspartate aminotransferase by $>25 \%$ from baseline, an increase in Child-Pugh score of $\geq 1$ from baseline, and the absence of radiological tumor response, may well predict refractoriness to TACE. Furthermore, an ART score of $\geq 2.5$ predicts a poor prognosis and the futility of further TACE sessions. ${ }^{11,12}$ However, increase in AST value (+25\%) from baseline had the highest importance (4 points), and the positivity of this parameter alone can lead the patient in the poor prognostic group. Recent studies conducted in Japan ${ }^{13}$ and Italy ${ }^{14}$ have failed to validate the ART score. In regard to the AST increase, it can be assumed that its role is milder in cases of viral cirrhosis than in alcoholic cirrhosis since it is a major cause in Austrian series, and AST tends to increase following toxic damage from alcohol. Alternative explanations are heterogeneous selection of intermediate-stage HCC or some differences in treatment techniques and time interval. For these reasons, several investigators in France reported on the ABCR scoring 
system, which contains four prognostic factors associated with OS, namely, BCLC and alpha-fetoprotein $(>200 \mathrm{ng} / \mathrm{mL})$ at baseline, an increase in Child-Pugh score of $>2$ from baseline, and the absence of radiological response. ABCR score from -3 to +6 was correlated with survival, and it has been demonstrated that an $\mathrm{ABCR}$ score of $>4$ prior to second TACE indicates a dismal prognosis and nonbenefit from further TACE. The ABCR score was validated in two different cohorts of 178 patients, and found to be better than the ART score in terms of predicting prognosis. ${ }^{15,16}$ Nevertheless, all these scoring systems should be tested in different populations and validated in prospective trials.

\section{Tumor response}

Traditionally, radiological response is measured in terms of tumor shrinkage using Response Evaluation Criteria in Solid Tumors (RECIST), which is based on the sum of unidimensional measurements of target lesions. However, the validity of the RECIST criteria has been challenged for assessing the radiological response to locoregional treatment of HCC, particularly TACE, which causes acute tumor necrosis that may not always be paralleled by a reduction in size. The EASL recommended bidimensionally measures change in the area of tumor enhancement on contrast-enhanced imaging. ${ }^{17}$ More recently, the American Association for the Study of Liver Diseases has proposed the modified RECIST (mRECIST) criteria to take into consideration changes in the degree of tumor arterial enhancement. ${ }^{18}$

Nowadays, the evaluation of tumor response after TACE is usually assessed using the mRECIST because the results of such evaluations are associated with clinical outcome and optimal treatment strategy. The recent clinical practice guidelines issued by EASL and European Organization for Research and Treatment of Cancer recommend that assessments of treatment response in HCC should be based on mRECIST criteria by contrast-enhanced CT or magnetic resonance imaging 4 weeks after initial therapy, ${ }^{3}$ and it has been demonstrated that mRECIST criteria are well correlated with survival. ${ }^{19,20}$ In one of these studies, 332 patients with intermediate-stage $\mathrm{HCC}$ and Child-Pugh A cirrhosis who underwent serial TACE were enrolled. All measurable target lesions of $\geq 1 \mathrm{~cm}$ in diameter were measured according to RECIST, EASL, and mRECIST, and both EASL and mRECIST criteria were found to be independent predictors of OS $(P<0.001) .{ }^{19}$ Furthermore, although mRECIST showed significant differences in probability of survival for all response categories $(P<0.001$ for each comparison), EASL criteria showed no significant difference in the probability of survival between partial response and stable disease categories $(P=0.71) .{ }^{19}$

\section{TACE vs DEB-TACE}

TACE was first used to treat HCC in $1974,{ }^{21}$ and was applied by Yamada et $\mathrm{al}^{22}$ in Japan to most unresectable HCCs using gelatin sponge particles and anticancer agents. In the 1990s, the selective retention of lipiodol within tumors was used to enhance therapeutic effects and increase chemotherapeutic exposure. ${ }^{5}$ Llovet et $\mathrm{al}^{23}$ demonstrated that TACE had survival benefits as compared with conservative treatment $(\mathrm{HR}=0.47$, $95 \% \mathrm{CI}=0.25-0.91, P=0.025)$ in their randomized controlled trials ( $\mathrm{RCT}$ ), and Lo et $\mathrm{al}^{24}$ reported that TACE resulted in marked tumor response and that 1-, 2-, and 3-year survival rates were significantly better in their TACE group (57\%, $31 \%$, and $26 \%$, respectively) than in controls $(32 \%, 11 \%$, and $3 \%$, respectively, $P=0.002$ ). Although only a limited number of RCTs have been conducted to determine the survival benefit of cTACE, a cumulative meta-analysis of studies has shown that 2-year survival of patients with unresectable HCC treated with cTACE is better than that of conservative management. ${ }^{25}$

DEB-TACE uses doxorubicin-loaded beads as a carrier to achieve local, sustained, and controlled release of chemotherapeutics and ischemic injury to the tumor. The clinical effects of DEB-TACE have been demonstrated by many RCTs. ${ }^{26,27}$ In a multicenter Phase II randomized trial including 201 European patients (PRECISION V) in 2010, it was found that DEB-TACE using DC-Beads had higher rates of complete response, objective response, and disease control than cTACE. ${ }^{26}$ However, there is still some debate about this study. Only a subgroup with Child-Pugh B, ECOG 1, bilobar disease, and recurrent disease showed a significant increase in objective response $(P=0.038)$ compared with cTACE. DEB-TACE was associated with improved tolerability, with a significant reduction in serious liver toxicity $(P<0.001)$ and a significantly lower rate of doxorubicin-related side effects $(P=0.0001)$.

Other studies have presented similar results. In a metaanalysis of nine studies (830 patients) conducted in 2014, DEB-TACE showed significantly better OS and progression free survival, and also higher objective response and disease control rates. ${ }^{2}$ However, in subgroup analyses, pooled results showed the survival benefits of DEB-TACE were not observed in prospective RCTs, but only in retrospective nonRCTs. ${ }^{2}$ Therefore, claims concerning the survival benefits of 
DEB-TACE vs cTACE should be interpreted with caution. Furthermore, the incidence of common adverse events such as nausea, pain, fever, and fatigue was not significantly different in the two groups.

\section{Combination strategies and synergies \\ TACE and molecular targeted therapy}

Although TACE is currently recommended as a first-line therapy for the treatment of intermediate-stage HCC, the incidence of recurrence is high, and multiple TACE sessions are needed to eradicate residual tumors. Furthermore, treatment with TACE can cause hypoxia and the release of factors that promote tumorigenesis, angiogenesis, and fibrosis. ${ }^{28}$ It has been shown that serum concentrations of vascular endothelial growth factor and fibroblast growth factor increase after TACE and that their serum levels are positively associated with the risk of disease progression, including tumor growth, recurrence, metastasis, and poor survival. ${ }^{28-30}$ The limited long-term benefit of TACE may be correlated with the activation of these proangiogenic factors. Sorafenib, as a multikinase inhibitor that targets vascular endothelial growth factor receptor, platelet-derived growth factor receptor, and Raf signaling, can block tumor growth and neoangiogenesis. ${ }^{11,31}$ As sorafenib targets TACE-induced angiogenic factors and potentially enhances its efficacy, ${ }^{12}$ the combination of sorafenib with TACE sounds reasonable and is considered to have the potential to improve the effectiveness of TACE.

Kudo et $\mathrm{al}^{32}$ conducted a RCT to evaluate the efficacy and safety of sorafenib after TACE in 458 Japanese and Korean patients with unresectable $\mathrm{HCC}$, who were randomized 1:1 to sorafenib $400 \mathrm{mg}$ bid or placebo, and showed sorafenib did not significantly prolong time to progression (TTP) (HR $=0.87,95 \% \mathrm{CI}=0.70-1.09, P=0.252)$ or $\mathrm{OS}(\mathrm{HR}=1.06$, $95 \% \mathrm{CI}=0.69-1.64, P=0.790)$. Another RCT found that in 80 hepatitis $\mathrm{C}$ virus (HCV)-infected patients with BCLC-B HCC undergoing TACE, median TTP was 9.2 months in the sorafenib-treated group and 4.9 months in the placebo-treated group $(\mathrm{HR}=2.5,95 \% \mathrm{CI}=1.66-7.56, P<0.001) .{ }^{33}$ However, the small sample size $(n=62)$ limited the evidence level. The SPACE study was a first global, Phase II, randomized, double-blind, placebo-controlled trial with a large sample size (154 vs 153) to evaluate the efficacy and safety of sorafenib plus DEB-TACE in patients with intermediatestage HCC. ${ }^{34}$ Although this study met the primary end point of TTP $(\mathrm{HR}=0.797, P=0.072$, and a one-sided $\alpha$ of 0.15$)$, it was just passable, and the intergroup TTP difference was not significantly satisfactory, which could have been due to the study design, as TACE treatments were performed on schedule and the patient population was heterogeneous. However, an Asia-Pacific subset analysis of the SPACE study showed that TTP and OS for DEB plus sorafenib were longer than those for DEB-TACE plus placebo. Meanwhile, Liu et $\mathrm{al}^{35}$ reported in a meta-analysis performed to assess the safety and efficacy of TACE plus sorafenib in patients with unresectable HCC that the HR for TTP was $0.76(95 \% \mathrm{CI}$ $=0.66-0.89, P<0.001)$ and the HR for OS was $0.81(95 \%$ $\mathrm{CI}=0.65-1.01, P=0.061)$. Accordingly, they proposed that combination therapy might have benefits in terms of TTP but not for OS.

Trials have also been conducted on combination of TACE with other agents such as brivanib, sunitinib, and thalidomide. In a Phase III study, TACE plus brivanib adjuvant therapy did not improve OS. ${ }^{36}$ A prospective RCT of TACE plus thalidomide in 108 patients with unresectable HCC clearly showed that combination treatment can postpone disease progression and prolong survival. ${ }^{37}$ Another propensity score matching study with sunitinib plus TACE for advanced-stage $\mathrm{HCC}$ suggests that combination therapy is superior to TACE alone in terms of OS and TTP. ${ }^{38}$

TACE plus molecular targeted therapy combinations are currently being evaluated in randomized trials. It is hoped that these ongoing trials will contribute to the determination of optimal combinations and timing, which will be crucial in improving outcomes with intermediate HCC.

\section{TACE and local ablative therapies}

Trials have also been conducted on TACE combined with local ablative therapies, but results were inconclusive. Gu et $\mathrm{al}^{39}$ undertook a meta-analysis to compare the effectiveness of TACE plus local ablative therapy and monotherapy in HCC. Eighteen studies were conducted involving 2,120 HCC patients treated with combination therapy or monotherapy (1,071 and 1,049 patients, respectively). The local therapies included were RFA, percutaneous ethanol injection, high-intensity ultrasound, percutaneous acetic acid, and cryoablation. The combination therapy group was found to have significantly better survival in terms of 1-, 2-, 3-, and 5-year survival rates (risk ratio [RR] 1.10, 95\% $\mathrm{CI}=1.03-1.18, P=0.005$; RR $1.20,95 \% \mathrm{CI}=1.10-1.30$, $P<0.0001$; RR 1.43, 95\% CI $=1.18-1.73, P<0.0001$; RR $1.40,95 \% \mathrm{CI}=1.22-1.61, P<0.0001$, respectively), OS (HR $0.66,95 \% \mathrm{CI}=0.51-0.85, P=0.001)$, and tumor response $(\mathrm{RR}$ $1.54,95 \% \mathrm{CI}=1.09-2.18, P=0.013)$ than the monotherapy group. According to the stratified analysis, some studies 
included actually compared TACE + RFA vs TACE, which could not support the use of RFA combination.

The combination of TACE and RFA has several theoretical advantages over RFA alone. Decreased blood flow induced by TACE reduces heat loss and increases the RFA ablative zone. Furthermore, lipiodol uptake after TACE makes ablative margins easier to evaluate. ${ }^{40}$ Thus, a combination of TACE and RFA would be expected to reduce local progression and improve overall and recurrence-free survival rates in patients with $\mathrm{HCC}^{40}$ In another metaanalysis undertaken to evaluate the clinical efficacy and safety of RFA combined with TACE and RFA monotherapy, TACE plus RFA was found to be more effective than RFA monotherapy, with significantly higher survival rates at 1 year $(8$ trials, $\mathrm{OR}=2.14,95 \% \mathrm{CI}=1.57-2.91 ; P<0.001)$, 3 years $(12$ trials, $\mathrm{OR}=1.98,95 \% \mathrm{CI}=1.28-3.07 ; P=0.001)$, and 5 years $(4$ trials, $\mathrm{OR}=2.70,95 \% \mathrm{CI}=1.42-5.14$; $P=0.003) .{ }^{40}$ Although RFA has been successfully used to treat small $(\leq 3 \mathrm{~cm}$ ) HCCs, the local tumor progression rate is higher for tumors that exceed $3 \mathrm{~cm}$ in diameter. ${ }^{41}$ Thus, tumor size is an important consideration in the choice of therapy. Shibata et $\mathrm{al}^{42}$ prospectively reported that the OS rate from combined TACE and RFA was equivalent to that of RFA alone in patients with resectable HCC with $\leq 3$ nodules smaller than $3 \mathrm{~cm}$, and they thought the combination of treatments may not be necessary for the treatment of small HCC. On the other hand, recent studies have indicated that combined TACE plus RFA is a safe and useful therapeutic option for medium-sized HCCs. ${ }^{43,44}$ Also, RCT conducted in 189 participants with HCC less than $7 \mathrm{~cm}$ (94 in the TACE plus RFA group and 95 in the RFA alone group) reported TACE plus RFA was superior to RFA alone in improving survival for patients with HCC less than $7 \mathrm{~cm} .{ }^{45}$

Although the role of combined RFA with TACE in the treatment of HCC has not been completely determined, these published results provide evidence of the rationale for using combination therapy in the future treatment of intermediatestage HCC.

\section{TACE and radiation therapy}

The meta-analysis of ten studies, which included 400 patients in the TACE plus Three-dimensional Conformal Radiation Therapy (3D-CRT) group and 508 patients in the TACE group, reported TACE plus 3D-CRT significantly improved 1 -year $(\mathrm{OR}=1.87,95 \% \mathrm{CI}=1.37-2.55, P<0.0001)$, 2-year $(\mathrm{OR}=2.38,95 \% \mathrm{CI}=1.78-3.17, P<0.00001)$, and 3 -year $(\mathrm{OR}$ $=2.97,95 \% \mathrm{CI}=2.10-4.21, P<0.00001)$ OSs. Furthermore, combination therapy resulted in higher complete and partial response rates $(\mathrm{OR}=3.81,95 \% \mathrm{CI}=2.70-5.37 ; P<0.00001)$ and a more rapid decline in serum alpha-fetoprotein levels (OR =3.24, 95\% CI =2.09-5.02, $P<0.00001)$. This metaanalysis indicated that TACE plus 3D-CRT achieved better results than TACE monotherapy, and the authors suggested that further large-scale multicenter trials be conducted. ${ }^{46}$

Technological advancements in radiation planning and delivery such as Stereotactic Body Radiotherapy (SBRT) are noninvasive, administered on an outpatient basis, and allow short treatment course and higher radiation dose to be focused on the target tumor while maximally sparing the surrounding noninvolved liver. A prospective Phase I trial of liver SBRT reported a 1 -year survival of $48 \%$ and that $29 \%$ of patients developed grade 3 or higher toxicity at 3 months. ${ }^{47}$ Another study of 17 HCC patients reported a 1-year survival of $77 \%$ and three cases of grade 3 toxicity at 1 year. ${ }^{48}$ Subsequently, a Phase II trial conducted to evaluate the efficacy of SBRT in 38 Child-Pugh class A patients with HCC found OS of $94 \%, 77 \%$, and $52 \%$ at 1,2 , and 3 years, respectively. ${ }^{36}$ On the basis of these results, TACE plus SBRT appears to be a promising effective combination therapy in unresectable HCC. Nevertheless, further studies are needed.

\section{Preoperative treatment}

TACE has also been used as a preoperative neoadjuvant chemotherapy to improve survival in patients with resectable HCC. ${ }^{49}$ However, several RCTs have reported no increase in survival for preoperative TACE. ${ }^{50-52}$ Nonetheless, TACE is the most frequently used therapy before liver transplantation for HCC, and is thus referred to as "bridging" therapy. ${ }^{53}$ However, relatively few prospective studies have been conducted on TACE before liver transplantation, and a study that compared cTACE and DEB-TACE showed no definite difference in local tumor control and OS. ${ }^{54}$

\section{Conclusion}

Here, we have provided a review of the literature on transcatheter arterial embolic therapies used to treat $\mathrm{HCC}$, but the level of evidence is generally not provided. Although TACE is the most commonly used first-line treatment for intermediatestage HCC and for some advanced-stage HCCs, its clinical benefits in advanced-stage HCC would undoubtedly be improved by a better understanding of the characteristics of HCC. Therefore, we have addressed the issues of cTACE, DEB-TACE, preoperative TACE, and several combined therapies with TACE, including sorafenib, RT, and RFA. The optimal treatment choice, in any given case, remains challenging because of the complexity of HCC and the 
need to balance treatment effectiveness and organ-function preservation, which requires an understanding of the characteristics of the HCC and the patient. In some cases, metaanalyses based on nonrandomized studies have been cited, and therefore conclusions could not be definitive before the results of randomized trials were available. In order to achieve better efficacy for treating patients with unresectable HCC, we still need new and combination approaches and further well-defined studies to develop future therapies.

\section{Acknowledgment}

This work was supported by an Inha University Research grant.

\section{Disclosure}

The authors report no conflicts of interest in this work.

\section{References}

1. Lencioni R, Petruzzi P, Crocetti L. Chemoembolization of hepatocellular carcinoma. Semin Intervent Radiol. 2013;30:3-11.

2. Zhou X, Tang Z, Wang J, et al. Doxorubicin-eluting beads versus conventional transarterialchemoembolization for the treatment of hepatocellular carcinoma: a meta-analysis. Int J Clin Exp Med. 2014;7: 3892-3903.

3. European Association for the Study of the Liver, European Organisation For Research, Treatment of Cancer. EASL-EORTC clinical practice guidelines: management of hepatocellular carcinoma. $J$ Hepatol. 2012;56:908-943.

4. Huang K, Zhou Q, Wang R, et al. Doxorubicin-eluting beads versus conventional transarterial chemoembolization for the treatment of hepatocellular carcinoma. J Gastroenterol Hepatol. 2014;29: 920-925.

5. Nishikawa H, Kita R, Kimura T, et al. Transcatheter arterial embolic therapies for hepatocellular carcinoma: a literature review. Anticancer Res. 2014;34:6877-6886.

6. Cheng AL, Kang YK, Chen Z, et al. Efficacy and safety of sorafenib in patients in the Asia-Pacific region with advanced hepatocellular carcinoma: a phase III randomised, double-blind, placebo-controlled trial. Lancet Oncol. 2009;10:25-34.

7. Xue TC, Xie XY, Zhang L, et al. Transarterial chemoembolization for hepatocellular carcinoma with portal vein tumor thrombus: a metaanalysis. BMC Gastroenterol. 2013;13:60.

8. Kim HY, Park JW, Joo J, et al. Severity and timing of progression predict refractoriness to transarterial chemoembolization in hepatocellular carcinoma. J Gastroenterol Hepatol. 2012;27:1051-1056.

9. Arizumi T, Ueshima K, Chishina $\mathrm{H}$, et al. Validation of the criteria of transcatheter arterial chemoembolization failure or refractoriness in patients with advanced hepatocellular carcinoma proposed by the LCSGJ. Oncology. 2014;87(Suppl 1):32-36.

10. Kudo M, Matsui O, Izumi, N. et al. JSH consensus-based clinical practice guidelines for the management of hepatocellular carcinoma: 2014 update by the liver cancer study group of Japan. Liver Cancer. 2014;3:458-468.

11. Raoul JL, Gilabert M, Piana G. How to define transarterial chemoembolization failure or refractoriness: a European perspective. Liver Cancer. 2014;3:119-124.

12. Hucke F, Sieghart W, Pinter M, et al. The ART-strategy: sequential assessment of the ART score predicts outcome of patients with hepatocellular carcinoma re-treated with TACE. J Hepatol. 2014;60: $118-126$.
13. Kudo M, Arizumi T, Ueshima K. Assessment for retreatment (ART) score for repeated transarterial chemoembolization in patients with hepatocellular carcinoma. Hepatology. 2014;59:2424-2425.

14. Sieghart W, Hucke F, Pinter M, et al. The ART of decision making: retreatment with transarterial chemoembolization in patients with hepatocellular carcinoma. Hepatology. 2013;57:2261-2273.

15. Adhoute X, Penaranda G, Naude S, et al. Retreatment with TACE: The ABCR SCORE, an aid to the decision-making process. J Hepatol. 2015; 62(4):855-862.

16. Adhoute X, Penaranda G, Raoul JL, et al. Retreatment with TACE: $\mathrm{ABCR}$ score and radiological response, a really tight connection. J Hepatol. 2015;62(6):1442-1443.

17. Bruix J, Sherman M, Llovet JM, et al. Clinical management of hepatocellular carcinoma. Conclusions of the Barcelona-2000 EASL conference. European Association for the Study of the Liver. J Hepatol. 2001;35:421-430.

18. Lencioni R, Llovet JM. Modified RECIST (mRECIST) assessment for hepatocellular carcinoma. Semin Liver Dis. 2010;30:52-60.

19. Shim JH, Lee HC, Kim SO, et al. Which response criteria best help predict survival of patients with hepatocellular carcinoma following chemoembolization? A validation study of old and new models. Radiology. 2012;262:708-718.

20. Gillmore R, Stuart S, Kirkwood A, et al. EASL and mRECIST responses are independent prognostic factors for survival in hepatocellular cancer patients treated with transarterial embolization. J Hepatol. 2011;55: 1309-1316.

21. Doyon D, Mouzon A, Jourde AM, et al. Hepatic, arterial embolization in patients with malignant liver tumours (author's transl). Ann Radiol. 1974; 17:593-603.

22. Yamada R, Nakatsuka H, Nakamura K, et al. Transcatheter arterial embolization therapy in unresectable hepatomas - experience in 15 cases. ACTA Hepatol Jpn. 1979;20:595-603.

23. Llovet JM, Real MI, Montana X, et al. Arterial embolisation or chemoembolisation versus symptomatic treatment in patients with unresectable hepatocellular carcinoma: a randomised controlled trial. Lancet. 2002;359:1734-1739.

24. Lo CM, Ngan H, Tso WK, et al. Randomized controlled trial of transarterial lipiodol chemoembolization for unresectable hepatocellular carcinoma. Hepatology. 2002;35:1164-1171.

25. Llovet JM, Bruix J. Systematic review of randomized trials for unresectable hepatocellular carcinoma: chemoembolization improves survival. Hepatology. 2003;37:429-442.

26. Lammer J, Malagari K, Vogl T, et al. Prospective randomized study of doxorubicin-eluting-bead embolization in the treatment of hepatocellular carcinoma: results of the PRECISION V study. Cardiovasc Intervent Radiol. 2010;33:41-52.

27. Sacco R, Bargellini I, Bertini M, et al. Conventional versus doxorubicineluting bead transarterial chemoembolization for hepatocellular carcinoma. J Vasc Interv Radiol. 2011;22:1545-1552.

28. Rosmorduc O, Housset C. Hypoxia: a link between fibrogenesis, angiogenesis, and carcinogenesis in liver disease. Semin Liver Dis. 2010;30:258-270

29. Xiong ZP, Yang SR, Liang ZY, et al. Association between vascular endothelial growth factor and metastasis after transcatheter arterial chemoembolization in patients with hepatocellular carcinoma. Hepatobiliary Pancreat Dis Int. 2004;3:386-390.

30. Schoenleber SJ, Kurtz DM, Talwalkar JA, et al. Prognostic role of vascular endothelial growth factor in hepatocellular carcinoma: systematic review and meta-analysis. Br J Cancer. 2009;100:1385-1392.

31. Chang YS, Adnane J, Trail PA, et al. Sorafenib (BAY 43-9006) inhibits tumor growth and vascularization and induces tumor apoptosis and hypoxia in RCC xenograft models. Cancer Chemother Pharmacol. 2007:59:561-574.

32. Kudo M, Imanaka K, Chida N, et al. Phase III study of sorafenib after transarterial chemoembolisation in Japanese and Korean patients with unresectable hepatocellular carcinoma. Eur J Cancer. 2011;47: $2117-2127$. 
33. Sansonno D, Lauletta G, Russi S, et al. Transarterial chemoembolization plus sorafenib: a sequential therapeutic scheme for HCV-related intermediate-stage hepatocellular carcinoma: a randomized clinical trial. Oncologist. 2012;17:359-366.

34. Lencioni R, Zou J, Leberre M. Sorafenib or placebo in combination with transarterial chemoembolization for intermediatestage hepatocellular carcinoma (SPACE). J Clin Oncol. 2010;28:(abstr TPS178).

35. Liu L, Chen H, Wang M, et al. Combination therapy of sorafenib and TACE for unresectable HCC: a systematic review and meta-analysis. PLoS One. 2014;9:e91124.

36. Kudo M, Han G, Finn RS, et al. Brivanib as adjuvant therapy to transarterial chemoembolization in patients with hepatocellular carcinoma: a randomized phase III trial. Hepatology. 2014;60:1697-1707.

37. Hao MZ, Lin HL, Chen Q, et al. Efficacy of transcatheter arterial chemoembolization combined thalidomide on hepatocellular carcinoma: a controlled randomized trial. Ai Zheng. 2007;26:861-865.

38. Chen J, Zhou C, Long Y, et al. Sunitinib combined with transarterial chemoembolization versus transarterial chemoembolization alone for advanced-stage hepatocellular carcinoma: a propensity score matching study. Tumour Biol. 2015;36:183-191.

39. Gu L, Liu H, Fan L, et al. Treatment outcomes of transcatheter arterial chemoembolization combined with local ablative therapy versus monotherapy in hepatocellular carcinoma: a meta-analysis. $J$ Cancer Res Clin Oncol. 2014;140:199-210.

40. Kong QF, Jiao JB, Chen QQ, et al. Comparative effectiveness of radiofrequency ablation with or without transarterial chemoembolization for hepatocellular carcinoma. Tumour Biol. 2014;35:2655-2659.

41. Llovet JM, Bruix J. Novel advancements in the management of hepatocellular carcinoma in 2008. J Hepatol. 2008;48(Supp1 1):S20-S37.

42. Shibata T, Isoda H, Hirokawa Y, et al. Small hepatocellular carcinoma is radiofrequency ablation combined with transcatheter arterial chemoembolization more effective than radiofrequency ablation alone for treatment? Radiology. 2009;252:905-913.

43. Yamakado K, Nakatsuka A, Ohmori S, et al. Radiofrequency ablation combined with chemoembolization in hepatocellular carcinoma: treatment response based on tumor size and morphology. JVasc Interv Radiol. 2002;13:1225-1232.
44. Takaki H, Yamakado K, Nakatsuka A, et al. Radiofrequency ablation combined with chemoembolization for the treatment of hepatocellular carcinomas $5 \mathrm{~cm}$ or smaller: risk factors for local tumor progression. J Vasc Interv Radiol. 2007;18:856-861.

45. Peng ZW, Zhang YJ, Chen MS, et al. Radiofrequency ablation with or without transcatheter arterial chemoembolization in the treatment of hepatocellular carcinoma: a prospective randomized trial. J Clin Oncol. 2013;31:426-432.

46. Zou LQ, Zhang BL, Chang Q, et al. 3D conformal radiotherapy combined with transcatheter arterial chemoembolization for hepatocellular carcinoma. World J Gastroenterol. 2014;20:17227-17234.

47. Tse RV, Hawkins M, Lockwood G, et al. Phase I study of individualized stereotactic body radiotherapy for hepatocellular carcinoma and intrahepatic cholangiocarcinoma. J Clin Oncol. 2008;26:657-664.

48. Price TR, Perkins SM, Sandrasegaran K, et al. Evaluation of response after stereotactic body radiotherapy for hepatocellular carcinoma. Cancer. 2012;118:3191-3198.

49. Nishikawa H, Arimoto A, Wakasa T, et al. Effect of transcatheter arterial chemoembolization prior to surgical resection for hepatocellular carcinoma. Int J Oncol. 2013;42:151-160.

50. Yamasaki $\mathrm{S}$, Hasegawa $\mathrm{H}, \mathrm{Kinoshita} H$, et al. A prospective randomized trial of the preventive effect of pre-operative transcatheter arterial embolization against recurrence of hepatocellular carcinoma. Jpn J Cancer Res. 1996;87:206-211.

51. Zhou WP, Lai EC, Li AJ, et al. A prospective, randomized, controlled trial of preoperative transarterial chemoembolization for resectable large hepatocellular carcinoma. Ann Surg. 2009;249:195-202.

52. Kaibori M, Tanigawa N, Kariya S, et al. A prospective randomized controlled trial of preoperative whole-liver chemolipiodolization for hepatocellular carcinoma. Dig Dis Sci. 2012;57:1404-1412.

53. Fujiki M, Aucejo F, Kim R. General overview of neo-adjuvant therapy for hepatocellular carcinoma before liver transplantation: necessity or option? Liver Int. 2011;31:1081-1089.

54. Frenette CT, Osorio RC, Stark J, et al. Conventional TACE and drug-eluting bead TACE as locoregional therapy before orthotopic liver transplantation: comparison of explant pathologic response. Transplantation. 2014;98:781-787.
Journal of Hepatocellular Carcinoma

\section{Publish your work in this journal}

The Journal of Hepatocellular Carcinoma is an international, peerreviewed, open access journal that offers a platform for the dissemination and study of clinical, translational and basic research findings in this rapidly developing field. Development in areas including, but not limited to, epidemiology, vaccination, hepatitis therapy, pathology and

\section{Dovepress}

molecular tumor classification and prognostication are all considered for publication. The manuscript management system is completely online and includes a very quick and fair peer-review system, which is all easy to use. Visit http://www.dovepress.com/testimonialsphp to read real quotes from published authors. 